\title{
Anisotropic Shift of the Irreversibility Line by Neutron Irradiation*
}

\author{
F. M. Sauerzopf, H. P. Wiesinger, and H. W. Weber \\ Atominstitut der österreichischen Universitäten, A-1020 Wien, Austria \\ G. W. Crabtree, M. C. Frischherz and M. A. Kirk \\ Materials Science Division, Argonne National Laboratory, Argonne, IL 60439
}

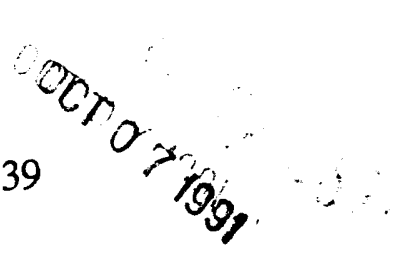

September 1991

\begin{tabular}{|c|}
\hline 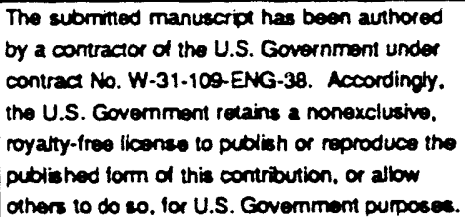 \\
\hline
\end{tabular}

\section{DISCLAIMER}

\begin{abstract}
This report was prepared as an account of work sponsored by an agency of the United States Government. Neither the United States Government nor any agency thereof, nor any of their employees, makes any warranty, express or implied, or assumes any legal liability or responsibility for the accuracy, completeness, or usefulness of any information, apparatus, product, or process disclosed, or represents that its use would not infringe privately owned rights. Reference herein to any specific commercial product, process, or service by trade name, trademark, manufacturer, or otherwise does not necessarily constitute or imply its endorsement, recommendation, or favoring by the United States Gowernment or any agency thereof. The views and opinions of authors expressed herein do not necessarily state or reflect those of the United States Government or any agency thereof.
\end{abstract}

Accepted for publication in Supercond. Sci. Technol. from the 6th International Workshop on Critical Currents and Superconductors, Cambridge, U.K., July 8-11, 1991.

*This work has been supported in part by Fonds zur Förderung der Wissenschafulichen Forschung, Wien, under contract \# 7970 and by the U. S. Department of Energy, RESMaterials Sciences, under contract W-31-109-Eng-38 (ANL). 
F. M. Sauerzopf, H. P. Wiesinger, and H W. Weber

Atominstitut der österreichischen Universitäten, A-1020 Wien, Austria

G. W. Crabtree, M. C. Frischherz, and M. A. Kirk

Materials Science Division, Argonne National Laboratory, Argonne, Il 60439, USA

\begin{abstract}
The ineversibility line of high- $T_{c}$ superconductors is shifted considerably by irradiating the material with fast neutrons. The anisotropic and non-monotonous shift is qualitatively explained by a simple model based on an interaction between three pinning mechanisms, the intrinsic pinning ij, the ab-planes, the weak pinning by the pre-irradiation defect structure, and strong pinning by neutron induced defect cascades. A correlation between the cascade density and the position of the irreversibility line is observed.
\end{abstract}

\title{
1. INTRODUCTION
}

The irreversibility line of high- $T_{c}$ superconductors has been the subject of intensive experimental work for some time (e. g. [1-3]). Many attempts to explain this transition from hysteretic to reversible magnetization behavior have been made (e. g. [1,4-8]), but the validity of these models is still under discussion [9], both because of simplifications in the models and the lack of sufficient accuracy and consistent interpretations of the experimental clata. On the other hand, some features of the irreversibility line are well established, e. $g$. the simple temperature dispendence of the imeversibility field, $\mathrm{H}^{*} \propto(1-\mathrm{T} / \mathrm{T})^{\mathrm{a}}$ with the exponent $\mathrm{a} \approx 1.5$ for $\mathrm{YBCO}$.

In this contribution we report on magnetization measurements on YBCO single crystals prior to and following fast neutron irradiation to various fluences. We will concentrate on the effect of fast neutron irradiation on the irreversibility line and present a model which contains the minimal requirements for a concept of flux pinning in these systems.

\section{EXPERIMENTAL}

In this set of neutron irradiation experiments a single crystal of YBCO [10] was irradiated to various fluences of fast neutrons up to $2 \times 10^{21} \mathrm{~m}^{-2}(\mathrm{E}>0.1 \mathrm{MeV})$. During irradiation the sample was kept in a helium atmosphere at about $50{ }^{\circ} \mathrm{C}$. The irreversibility line was determined from both magnetization curves at fixed termperatures and $\mathrm{zfc}-\mathrm{fc}$ measurements at various fields in a SQUID magnetometer. To obtain information on the anisotropy of the effect, measurements with the external field $\mathrm{H}_{\mathrm{a}}$ parallel to $\mathrm{c}$ as well as parallel to the ab-planes were made. Unfortumately the irreversibility line is not an intrinsic material parameter, but varies to a large extent with experimental conditions. In order to compare values deduced from different experiments, the time scale as well as the driving force on the flux lines and the resolution of the experiment have to be specified. In our experiment we do not evaluate the irreversibility line from the closing of the magnetization- or $\mathrm{fc}$ - and zfc-curves, but use the specific measuring process of the magnetometer for that purpose. In the magnetometer the sample is moved through the pick-up coils in a 
slightly inhomogeneous field ( $\delta \mathrm{H} \triangleleft 0.1 \%$ of $\mathrm{H}_{2}$ ), which is equivalent to the superposition of a small low-frequency ac-field onto the dc-field. This ripple field causes characteristic distortions of the response curves, if flux pinning is present. The absence of these distortions is clear evidence for the reversibility of the magnetization on the time scale of the movement $(\delta \mathrm{t}=35 \mathrm{~s})$ and within a resolution of $\mathrm{J}_{\mathrm{c}}<10^{4}-10^{5} \mathrm{Am}^{-2}$.

The results of these experiments are shown in figure 1 . The effect of neutron irradiation on the irreversibility lines is highly anisotropic and the shift of the lines with increasing neutron fluence is non-monotonous. For $\mathrm{H} \| \mathrm{c}$, the inreversibility line is shifted to lower temperatures at the first irradiation $\operatorname{step}\left(5 \times 10^{20} \mathrm{~m}^{-2}\right)$ and subsequently raised again. At a fluence of $2 \times 10^{21} \mathrm{~m}^{-2}$ we find about the same results as in the unirradiated state. The agreement with a similar experiment on oriented grained YBCO [11], where measurements on the unirradiated sample as well as at a fluence of $\sim 2 \times 10^{21} \mathrm{~m}^{-2}$ were made, is poor. This discrepancy will be discussed in the next chapter.

For $\mathrm{H}_{\mathrm{a}} \| \mathrm{ab}$, we observe a completely different behavior. In this case, the irreversibility line is shifted to much higher values following irradiation to the lowest neutron fluence, but remains almost unaffected at higher fluences except for a small decrease at the highest fluence of $2 \times 10^{21} \mathrm{~m}^{-2}$. A comparable overall shift towands $T_{c}$ has also been observed in [11].

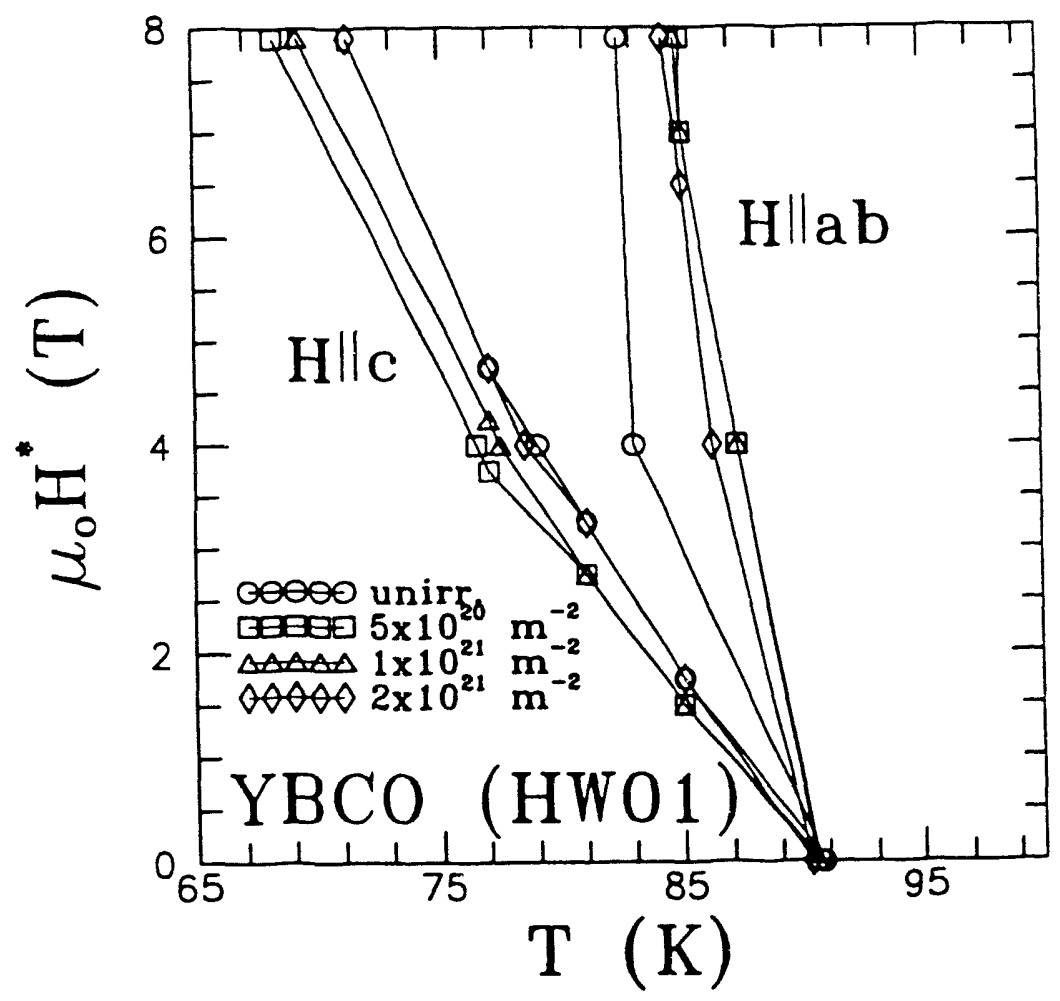

Figure 1. Irreversibility lines as a function of neut in fluence for $\mathrm{H} \| \mathrm{c}$ and $\mathrm{H} \| \mathrm{ab}$, respectively.

It has to be noted, that previous experiments on YBCO single crystals subjected to $3 \mathrm{MeV}$ proton irradiation [12], and on polycrystals irradiated with $25 \mathrm{MeV}$ electrons [13] have led to completely different results. As will be seen in the next chapter, this may be attributed to the different defect sizes and concentrations prevailing under various experimental conditions.

\section{PINNING MODEL}

The non-monotonous effect of neutron irradiation on the critical current densities of YBCO single crystals has already led to the assumption of an interaction between the pre-irradiation defect structure and the neutron induced defect cascades [14]. One important factor for the pinning structure in YBCO is the inherent oxygen deficiency of the crystals. Thus many oxygen deficient point defects exist, which will be quite mobile at room temperature and therefore accumulate at defect sinks in the crystal, where they presumably pin flux lines collectively. During neutron irradiation at slightly elevated temperatures, defect cascades are introduced into the material. At the time of the creation of a cascade, oxygen being the lightest atom in the lattice is removed from many positions within the cascade and recombines only partly at the previous sites. This leads to an oxygen movement in the crystal. It will 
again be collected at defect sinks, which leads to a recombination with oxygen defects already existing at these sites. Thus, by neutron irradiation the pre-existing defect structure may be weakened significantly. This can explain the downward-shift of the imeversibility line for $\mathrm{H} \| \mathrm{c}$ at the lowest neutron fluence. If oxygen defects do not play an important role, which could be the case in the textured samples of [11], this downward shift will not oocur and only a shift towards $T_{c}$ will be observad due to the introduction of the strong pinning centers. The model is supported by the actual data, since the minima and the maxima of the irreversibility lines compare favorably between the two experiments. The assumption of a weakening of the pre-irradiation defect structure in single crystals is also strongly supported by the measurements reported in ref. [15].

After irradiation, the resulting defect cascades will certainly lead to strong flux pinning. The mean diameter of a cascade $d_{\text {def }}$ is about $5 \mathrm{~nm}$ [10, which is close to the flux line diameter of $2 \xi_{\mathrm{ab}} \approx 7.6 \mathrm{~nm}$ at $77 \mathrm{~K}$. Smaller defect diameters may drastically affect the elementary pinning forces (cf. [13]), which can result in negligible shifts of the irreversibility line although $\mathrm{J}_{\mathrm{c}}$ may increase considerably.

The role of strong pinning centers may be seen more clearly from another geometrical argument. In the vicinity of the irreversibility line we do not expect a strong correlation between the flux lines (the shear modulus of the flux line lattice is negligible). Thus, only one flux line may be pinned by one defect of a comparable size, while other flux lines may easily flow by. Therefore a significant pinning effect of even the strongest pinning centers on the irreversibility line may only be expected at sufficiently high defect concentrations. Furthermore, the flux line lattice spacing a can be compared with the mean distance between the defects $a_{\text {def }}$ which could lead to a peak effect in the irversibility line when $a_{0}=a_{\text {def }}$ TEM [16 on a crystal irradiated to a neutron fluence of $2 \times 10^{21} \mathrm{~m}^{-2}$, has shown that the defect density at this fluence was $10^{22} \mathrm{~m}^{-3}$. Hence, $a_{\text {def }}$ is about $46 \mathrm{~nm}$ and the "matching" lattice parameter a 0 corresponds to a field of $-0.9 \mathrm{~T}$. This neutron fluence is the highest of our present series of irradiations and, hence, the defect spacing is always larger than $a_{0}$ for fields above $1 \mathrm{~T}$. Since lower fields have not been investigated unfortumately, a new series of experiments will be needed to clarify this point for $\mathrm{H} \| \mathrm{c}$. Some indications of this trend can be seen by replotting the irreversibility lines as shown in figure 2 . It will be noted that the irreversibility line for $\mathrm{H} \| \mathrm{c}$ starts to deviate from the otherwise uniform temperature dependence at low fields (arrow 1).

For $\mathrm{H}_{\mathrm{a}} \| \mathrm{ab}$ the influence of intrinsic pinning by the $\mathrm{Cu}-\mathrm{O}$ planes [17] has to be considered, which modifies our model significantly. If this pinning mechanism is strong enough, flux lines are one-dimensionally confined to their planes. Consequently, one large defect will be able to block about four of these tracks, and the flux lines following behind the pinned one in the same track, will not be able to flow by this point due to their one-dimensional confinement. Thus we have to replace $a_{\text {def }}$ by $a_{\text {def }} / 2-d_{\text {def }}$ This leads to a much higher matching field for a given

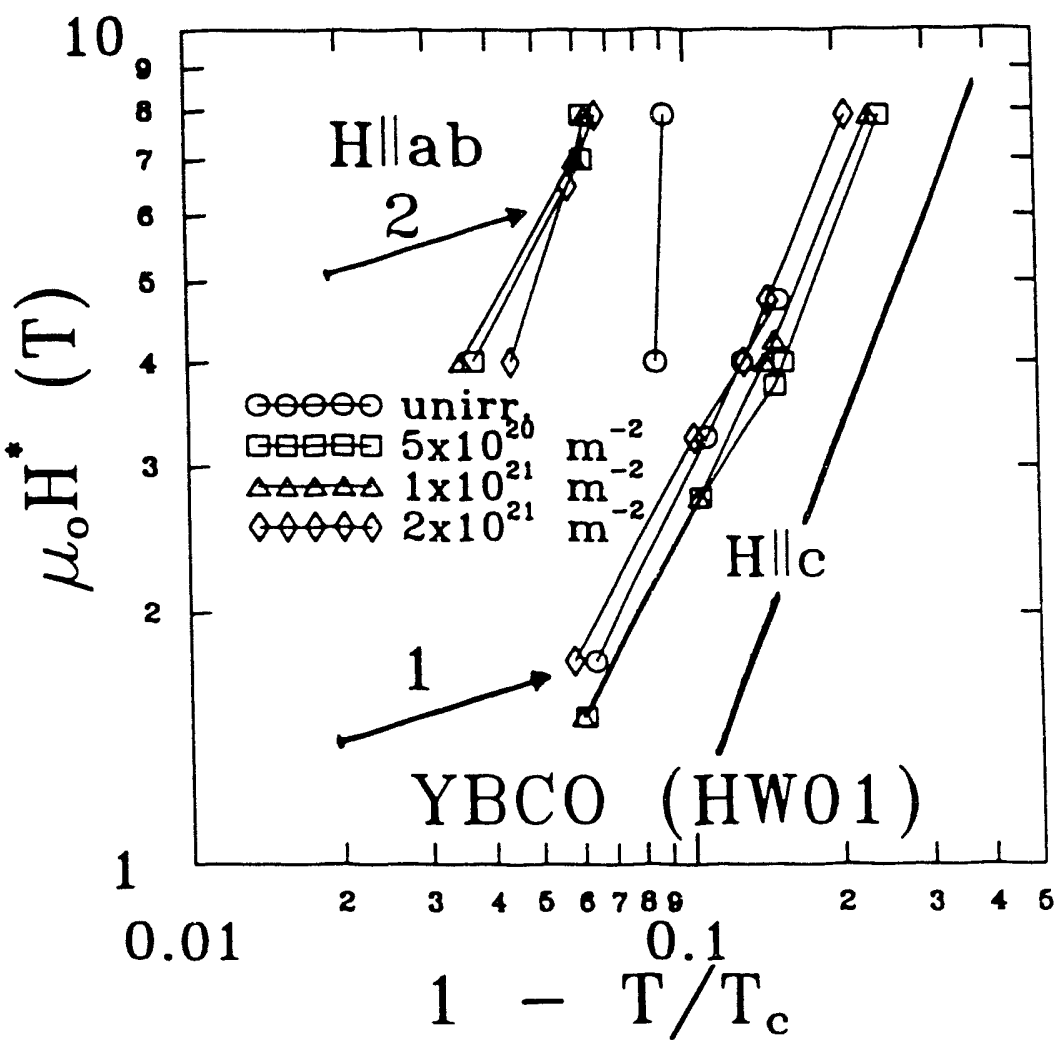

Figure 2. Irreversibility lines from figure 1, replotted on a logarithmic scale. The solid line represents the power law for $\mathrm{H}^{*}$ with $\mathrm{a}=1.5$. 
defect density (6 T at $2 \times 10^{21} \mathrm{~m}^{-2}$, as indicated by arrow 2 in figure 2). The lowest neutron fluence is already sufficient to shift the irreversibility line to very high values. Here the hysteretic region is limited by the intrinsic pinning. Therefore the irreversibility line is not affected much by the following irradiation steps and the peaks at the matching fields are very poorly developed. At higher fluences we expect the irradiation to gradually eliminate intrinsic pinning and, hence, to shift the irreversibility line towards the results for $\mathrm{H} \| \mathrm{c}$, as has been observed on YBCO films [18].

\section{CONCLUSIONS}

The experimental results on the anisotropy of the irreversibility lines as well as their different response to neutron irradiation has been discussed in terms of at least three different pinning mechanisms. Radiation induced changes of these mechanisms are attributed to a weakening of the pre-irradiation defect structure, the introduction of strong pinning centers and the gradual elimination of intrinsic pinning for $\mathrm{H} \|$ ab. Similar work on BiSCCO is under way [19], but cannot be analyzed yet within this model because of the lack of high fluence data.

\section{ACKNOWLEDGEMENTS}

This work has been supported in part by Fonds zarr Fördenung der Wissenschaftichen Forschung, Wien, under contract \# 7970 and by the U.S. Department of Energy, BES-Materials Science, under contract \# W-31-109-ENG38 (GWC). We gratefully acknowledge valuable help by W. Kritscha, $H$. Niedermaier, E. Klapfer and H. Schachner (Wien).

\section{REFERENCES}

[1] Yeshurun Y and Malozemoff A P 1988 Phys. Rev. Lett. 60 2202-2205

[2] Neumann C, Ziemann P and Geerk J 1989 Europhys. Lett. $10771-776$

[3] Kritscha W, Sauerzopf F M, Weber H W, Craburee G W, Chang Y C and Jiang P Z 1991 Physica C in print

[4] Clem J R 1988 Physica C 153-155 50-55

[5] Matsushita T, Fujjyoshi T, Toko K and Yamafuji K 1990 Appl. Phys. Lett. $562039-2041$

[6] Kes P H, Aarts J, van den Berg J, van der Beek C J, and Mydosh J A 1989 Supercond. Sci. Technol. 1242

[7] Griessen R 1991 Physica C 175 315-323

[8] Geshkenbein V B, Vinokur V M, Fehrenbacher R 1990 preprint

[9] Salem-Suqui S Jr, Shi D and McFarland S E 1991 preprint

[10] Kaiser D L, Holtzberg F, Chisholm M F and Worthington T K 1987 J. Cryst. Growth 85 593-598

[11] Küpfer H, Keller C, Meier-Himer R, Salama K, Selvamanickam V and Tartaglia G P 1990 preprint

[12] Civale L, Marwick A D, McElfresh M W, Worthington T K, Malozermoff A P, Holtzberg F H, Thompson J R and Kirk M A 1990 Phys. Rev. Letr. 651164

[13] Koncykowski M, Rullier-Albenque F, Yeshurun Y, Yacoby E R, Shaulov A and Gilchrist J 1991 Supercond.

Sci. Technol. 4 445-447

[14] Sauerzopf F M, Wiesinger H P, Kritscha W, Weber H W, Crabtree G W and Liu J Z 1991 Phys. Rev. B 43 $3091-3100$

[15] Lensink J G, Griessen R, Wiesinger H P, Sauerzopf F M, Weber H W and Crabtree G W 1991 this volume

[16] Frischherz M C 1991 thesis, to be published

[17] Tachiki M and Takahashi S 1989 Solid State Comm. 70 291-295

[18] Schalk R M, Weber H W, Barber Z H, Davies C E, Evetts J E, Somekh R E and Kim D H 1991 this volume

[19] Kritscha W, Sauerzopf F M, Weber H W, Crabtree G W, Chang Y C and Jiang P Z 1991, thics volume 

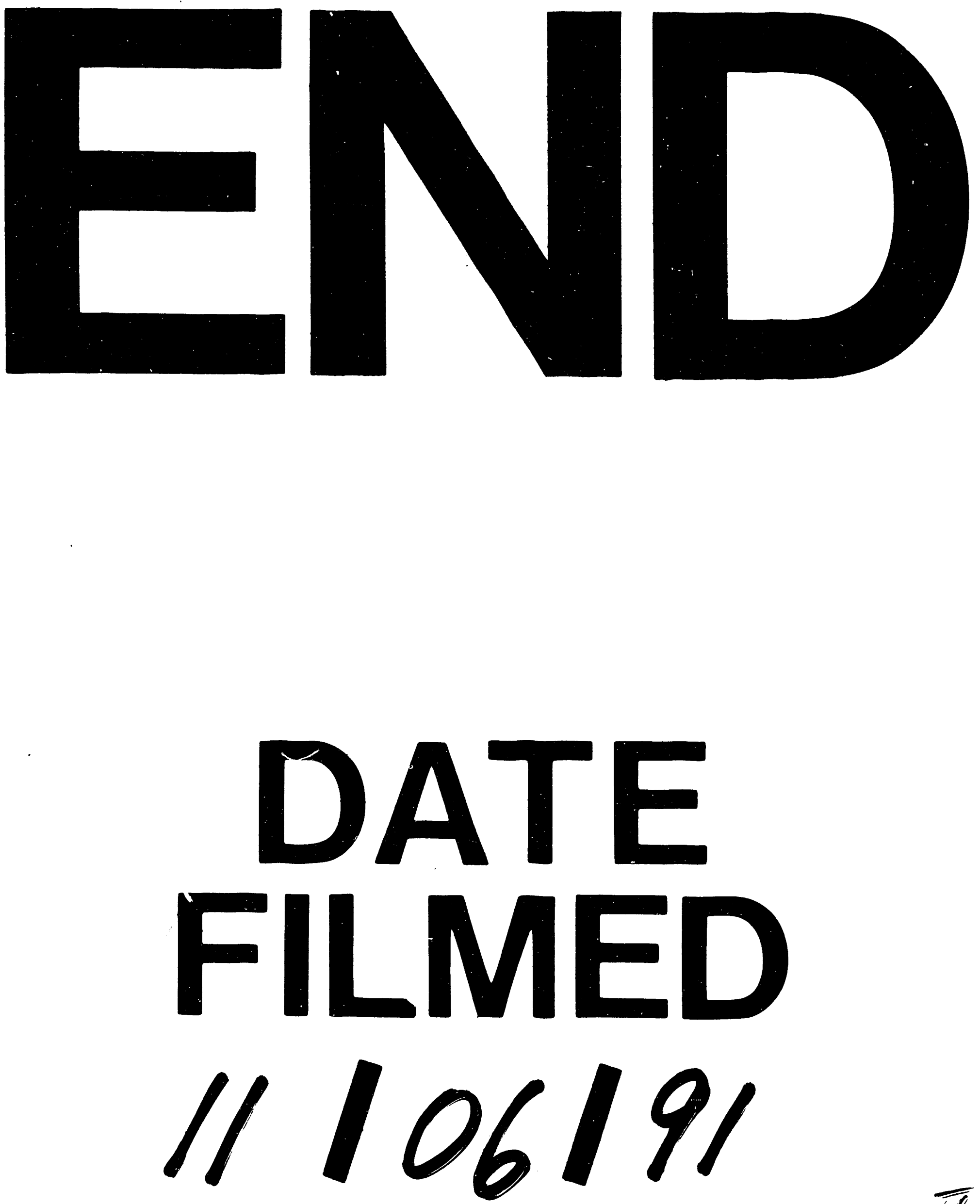

$\bar{T}$ 
